\title{
Editorial: Second Line Treatment of Non-Small Cell Lung Cancer: Clinical, Pathological and Molecular Aspects of Novel Promising Drugs
}

\author{
Umberto Malapelle ${ }^{1 * t}$ and Pierlorenzo Pallante ${ }^{2 *+}$ \\ ${ }^{1}$ Department of Public Health, University of Naples Federico II, Naples, Italy, ${ }^{2}$ Institute of Experimental Endocrinology and \\ Oncology (IEOS) "G. Salvatore", National Research Council (CNR), Naples, Italy
}

Keywords: NSCLC, liquid biopsy diagnostics, precision medicine, molecular markers, immunotherapy, antiangiogenic therapy

Editorial on the Research Topic

Second Line Treatment of Non-Small Cell Lung Cancer: Clinical, Pathological and Molecular Aspects of Novel Promising Drugs

The advent of precision medicine and predictive molecular pathology led to a revolution in clinical management of patients with non-small cell lung cancer. The discovery of oncogene addiction allowed the development of targeted therapies that represent newer therapeutic options reserved to those patients harboring specific gene alterations, such as EGFR mutations, ALK, and ROS1 translocations (Lazzari et al.; Sullivan and Planchard; Tran and Klempner; Köhler). In addition, the

OPEN ACCESS

Edited and Reviewed by: Luigi M. Terracciano, University of Basel, Switzerland

*Correspondence: Umberto Malapelle umberto.malapelle@unina.it; Pierlorenzo Pallante pallante@ieos.cnr.it

tThese authors have contributed equally to this work.

Specialty section: This article was submitted to Pathology,

a section of the journa

Frontiers in Medicine

Received: 14 April 2017 Accepted: 04 May 2017

Published: 22 May 2017

Citation: Malapelle U and Pallante P (2017) Editorial: Second Line Treatment of Non-Small Cell Lung Cancer: Clinical, Pathological and Molecular Aspects of Novel Promising Drugs. Front. Med. 4:63.

doi: 10.3389/fmed.2017.00063 introduction of immunotherapy, with anti PD-1 Pembrolizumab, in first-line treatment of NSCLC represents the best choice for EGFR, ALK, and ROS1 wild-type patients expressing PD-L1 on $\geq 50 \%$ of neoplastic cells (Cortinovis et al.). Despite the survival improvement achieved with these new therapeutic options in first-line treatment, about $30 \%$ of patients do not obtain a tumor response (Lazzari et al.). Moreover, those patients, initially sensitive to these treatments, acquire resistance and develop tumor progression. Approximately $60 \%$ of the patients progressing from first-line therapy receiving further systemic treatment in the second-line setting (Lazzari et al.; Cortinovis et al.) Also in second line, the armamentarium for the treatment of patients with NSCLC, includes a pletora of new drugs, such as immune checkpoint inhibitors (Pembrolizumab, Nivolumab) (Cortinovis et al.), third generation tyrosine kinase inhibitors (Osimertinib) (Molina-Vila et al.; Zugazagoitia et al.), and anti-angiogenic agents (Nintedanib and Ramucirumab) (Corrales et al.; Manzo et al.).

This exciting therapeutic scenario for NSCLC patients still has unsolved questions and challenging issues, in particular regarding the optimal selection of the patient population through the individualization of the correct methodology and biological source of material (tissues vs liquid biopsy) for clinical relevant biomarkers assessment (Molina-Vila et al.). Probably the right way is to give all the available opportunities to patients, but challenges and pitfalls should be carefully debated.

Taken together, the papers published in Research Topic "Second Line Treatment of Non-Small Cell Lung Cancer: Clinical, Pathological and Molecular Aspects of Novel Promising Drugs" represent a critical discussion focused on the older therapies and the historical development of second line, putting into perspective the new agents available in clinical practice, defining their importance from a clinical point of view, but also to consider and exploit the complex molecular mechanisms responsible of their efficacy or of the subsequently observed resistance phenomena, to support the oncologist to design the best therapeutic strategies for NSCLC patients.

\section{AUTHOR CONTRIBUTIONS}

$\mathrm{UM}$ and PP contributed equally to this paper. 
Conflict of Interest Statement: The authors declare that the research was conducted in the absence of any commercial or financial relationships that could be construed as a potential conflict of interest.

Copyright (c) 2017 Malapelle and Pallante. This is an open-access article distributed under the terms of the Creative Commons Attribution License (CC BY).
The use, distribution or reproduction in other forums is permitted, provided the original author(s) or licensor are credited and that the original publication in this journal is cited, in accordance with accepted academic practice. No use, distribution or reproduction is permitted which does not comply with these terms. 